\title{
Depressive and Anxious Adolescents: Do They Profit from Psychodynamic Therapy?
}

\author{
Inge Seiffge-Krenke \\ Department of Psychology, University of Mainz, Mainz, Germany \\ Email: seiffge-krenke @uni-mainz.de
}

How to cite this paper: Seiffge-Krenke, I (2020). Depressive and Anxious Adolescents: Do They Profit from Psychodynamic Therapy? Psychology, 11, 563-571. https://doi.org/10.4236/psych.2020.114037

Received: January 30, 2020

Accepted: March 29, 2020

Published: April 2, 2020

Copyright $\odot 2020$ by author(s) and Scientific Research Publishing Inc. This work is licensed under the Creative Commons Attribution International License (CC BY 4.0).

http://creativecommons.org/licenses/by/4.0/

\begin{abstract}
The purpose of this study was to determine the impact of psychodynamic treatment in reducing symptoms in depressive and anxious adolescents. In a 3 -wave longitudinal study, a sample of 303 adolescent patients in treatment was investigated. Their parents filled in a questionnaire (CBCL) in order to assess symptomatology (anxiety and depression) at three waves (beginning, middle and end of therapy) with approximately 1 year in between. The results clearly demonstrate that psychodynamic treatment showed a moderate to high effect in reducing symptomatology. For depression, the effect ranged between $\eta^{2}=0.40$ and $\eta^{2}=0.71$; for anxiety, the effect ranged between $\eta^{2}=$ 0.61 and $\eta^{2}=0.78$. Anxiety symptoms of the adolescent patients were reduced especially in the later course of the therapy.
\end{abstract}

\section{Keywords}

Psychodynamic Therapy, Adolescent Patients, Depression, Anxiety, Parents' Report

\section{Introduction}

The prevalence rate for major depression in adolescents ranges between 0.4 and 6.4\% (Benjet, Georgiades, \& Swendsen, 2010), whereas the prevalence of anxiety disorders in adolescents varies between $10 \%$ and $31.9 \%$ (Merikangas et al., 2010). While there have been no gender differences referring to depression during childhood, adolescent populations show that girls are being diagnosed two to three times more often than boys. The prevalence rates of depressive disorders approach the frequencies found for adulthood, and from adolescence onwards, the gender differences also correspond to those of adulthood.

It is unclear to what extent childhood depression is a precursor to adolescent depression. We assume that children, on a genetic basis, may be more or less susceptible to respond depressively when they have to cope with loss and stress 
(Costello, Foley, \& Angold, 2006). For example, the risk of developing depression in the presence of a serious stressor is 2.4 times higher for adolescents with a high genetic risk than for youth with a low risk. However, many constitutionally depressed children who stood out in their family by their separation anxiety, listlessness and their moods throughout childhood can suddenly become independent and self-initiating as adolescents. Noteworthy, there are also numerous symptoms of anxiety and depression on the subclinical level. Depressed mood (i.e., a sad, joyless and reluctant mood) over an unspecific period is reported by $30 \%$ to $70 \%$ of a non-clinical sample (Polanczyk, Salum, Sugaya, Caye, \& Rohde, 2015). Such depressive mood is obviously the rule rather than the exception if it occurs over a limited period.

In this study we focus on clinically relevant depressive and anxious symptoms, referring to the ICD-11 diagnosis of F32 and F40, which seemed to exist frequently together in one patient (Ezpelata \& Domènech, 2006). Pure depression is rather an exception in adolescence. Hinden, Compas, Howell, \& Achenbach (1997) report the highest comorbidity rates between depressive disorders and anxiety disorders, while there are lower comorbidities between depression and substance or drug abuse and eating disorders.

The question "Is treatment working?" (Liu \& Adrian, 2019), is an important one, as untreated depression and anxiety disorders show high stability over time, bear enormous suffering for the patients, and result in a high economic burden of untreated psychopathology for future health systems, including social and educational impairment. This indicates the urgent need for studies of the effectiveness of psychodynamic therapy in adolescents with anxiety and depression.

Although research on psychotherapeutic treatment in childhood and adolescence has increased significantly in recent decades, it is still well behind research on psychodynamic treatment in adulthood (see Chorpita et al., 2011). In particular, studies to verify the effectiveness of psychodynamic therapy in adolescents have been underrepresented. Weisz et al. (2005) demonstrated in a review that, of the controlled psychotherapy studies published on children and adolescents, only $1.7 \%$ had studied a psychodynamic treatment approach, although psychodynamic procedures were among the most commonly used in children and adolescents (Weisz \& Jensen, 2001).

Compared to the large number of studies demonstrating positive outcomes of cognitive-behavioral psychotherapy for different disorders (see, for example, Shirk et al., 2009; Szigethy et al., 2007; Herbert et al., 2009), the small number of efficacy studies in psychodynamic therapy including adolescent samples is striking (Fonagy, Roth, \& Higgitt, 2005; McCarty \& Weisz, 2007). In a recent meta-analysis (Weisz et al., 2017), only one third of the sample were adolescent patients and only 29 of the reviewed 447 studies included have investigated the effects of psychodynamic treatment.

\section{Research Questions}

In this research, we focus on the efficacy of psychodynamic treatment in reduc- 
ing adolescent patients' symptomatology, with a focus on depression and anxiety. Two research questions are guiding our study:

1) Does psychodynamic therapy work in adolescent outpatients and lead to a substantial reduction of depression and anxiety symptoms?

2) Is the change in anxiety and depression symptoms similar or different over time?

We will answer these questions by using a longitudinal design, in which we followed 303 adolescent patients. Assessments of the adolescents' symptomatology were done by their parents at three times during treatment: Time 1, beginning of treatment, Time 2, middle of treatment and Time 3, end of treatment.

\section{Method}

\subsection{Adolescent Patients and their Parents}

The treatment sample consisted of 303 adolescent patients and their respective parents. Patients were recruited in a large in-patient unit of a university town. The sample included only adolescent patients (12 - 18 years) who were diagnosed with clinically relevant symptoms according to ICD-11. Excluded were patients who, additionally, showed neurological abnormalities or had organic disease. The screened patients took part in psychodynamic treatment (1 hour per week) over three measurement times (beginning, middle and end of the therapy) with an average treatment duration of 85.9 hours. The average age at Time 1 (beginning of treatment) was 12.06 years ( $S D=2.86$ years); at Time 3 (end of treatment), the mean age was $M=15.1$ years; $S D=1.25$ ). Of the adolescent patients, $53.1 \%$ were female and $46.9 \%$ were male. Most of them (84.5\%) were German nationals.

During the treatment phase, parents filled in the assessments at the three measurement times. Parents' report of the child's symptomatology was given by 70 fathers and 233 mothers. $20.1 \%$ of the fathers had a secondary school education, $22.4 \%$ had a middle school education and $23.1 \%$ had a high school diploma; the percentages are roughly comparable for the mothers. Only $3.3 \%$ of mothers and fathers had no school leaving certificate. $43.9 \%$ of the patients lived in two-parent families, 33\% lived in single-parent families, and $23.1 \%$ lived in a different family form (including stepfamilies, foster families, and residential homes). The average number of siblings was 1.67.

\subsection{Measures}

Diagnosis by ICD-11. In order to establish a diagnosis, the adolescents were seen by an independent researcher. Each adolescent received a clinical diagnosis, based on the criteria established in the ICD-11. Of the total sample, 42 patients received the diagnosis F32 and 20 patients received the diagnosis F40. In the total sample, $22 \%$ of the patients who had been diagnosed as depressed also received a diagnosis as being anxious. In this study, we analyzed the data of patients receiving either anxiety or depression as a diagnosis. Therefore, patients 
suffering from both diagnoses (F32 and F40) were excluded from the study.

Parents' report of their child's psychopathology. Parents rated their child's symptomatology by completing a German translation of the Child Behavior Checklist (CBCL, Achenbach \& Edelbrock, 1983; Döpfner, Berner, \& Lehmkuhl, 1995). This instrument consists of 112 items that measure symptoms, by using an answer format of $0=$ not true, $1=$ somewhat or sometimes true, and $2=o f$ ten or very often true. The CBCL includes broadband syndromes of internalizing symptoms (e.g. example anxious, depressed, withdrawn) and externalizing (.g. delinquent, aggressive). We used the subscale anxious and depressed of the CBCL in this study. Cronbach's alphas for the score of parents' ratings of their child's psychopathology on being depressed or anxious across all 3 waves ranged from 0.81 to 0.89 , respectively. Norms, reliability and validity of the German versions of the CBCL are well-established (Döpfner et al., 1995).

\subsection{Procedure}

In the treatment sample, the CBCL was assessed at the beginning, in the middle phase and at the end of psychodynamic treatment with approximately 1 year in between. The parents of all participants provided written informed consent.

\subsection{Plan of Analysis}

All calculations were performed using the software $\mathrm{IBM}^{\circledR}{ }^{\circledR}$ SPS ${ }^{\circledast}$ Statistics 25 . The tests were carried out at a significance level of $\alpha=0.05$. In order to investigate whether there are diagnosis-dependent differences in symptom reduction over the course of therapy, an analysis of variance with repeated measurements was calculated for the F32 and F40 diagnosis group (repeated measures ANOVA). Parents' report of the CBCL at three measurement times was used as a measure of the anxious and depressed symptomatology over time. In the following, pairwise comparisons (with adjustments for multiple comparisons according to Bonferroni) were calculated in order to identify between which measurement times significant changes occurred.

\section{Results}

Figure 1 presents the mean values of CBCL depressed and anxious subscale separately for adolescents diagnosed with F32 and F40.

For the diagnostic group F32 depression, parents reported a significant decrease in symptom burden over time. The calculations based on the CBCL values showed both a significant change in the depressed subscale, $\mathrm{F}(2,40)=100.50, p$ $<0.001, \eta^{2}=0.710$, and the anxious subscale, $\mathrm{F}(2,40)=27.06, p<0.001, \eta^{2}=$ 0.398 . The pairwise comparisons showed significant reductions from Time 1 to Time 2, Time 1 to Time 3 and Time 2 to Time 3 for the depressive symptoms (all $p<0.001)$. For anxiety symptoms, the reduction was also significant between Time 1 and Time 3 and Time 2 and Time 3 (both $p<0.001$ ), but there was no significant change between Time 1 and Time 2. 


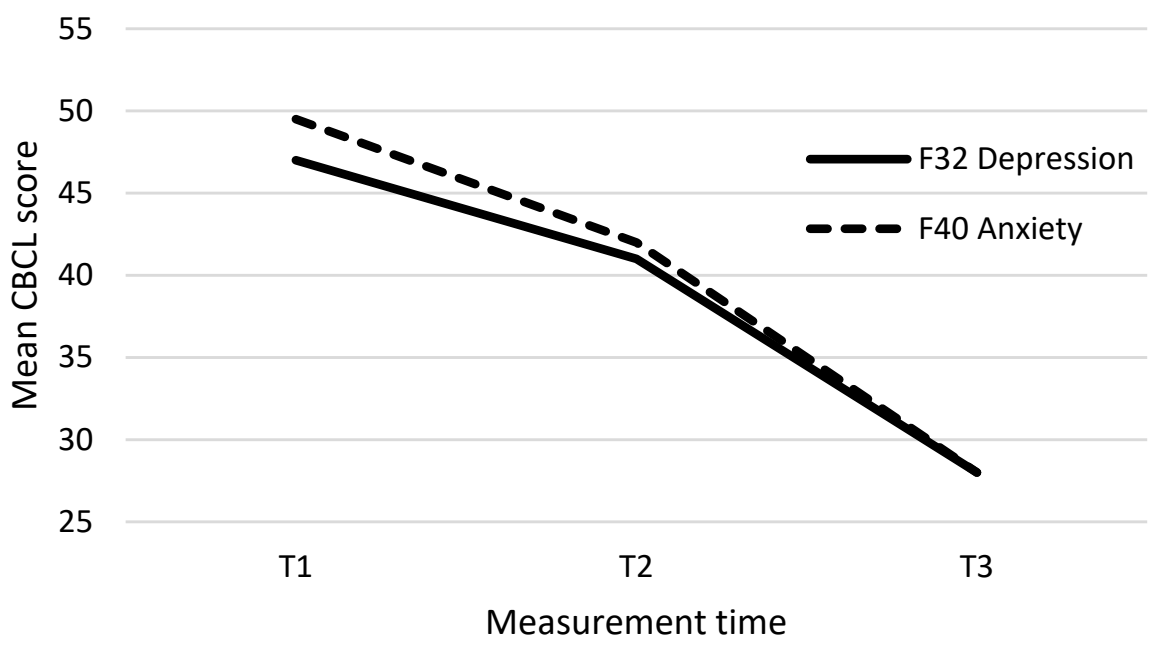

Figure 1. Parents' report of changes in depressive and anxious symptoms in adolescents suffering from clinical depression (F32) and anxiety disorders (F40).

For the diagnosis group F40 anxiety disorders, there were significant symptom reductions on the depressed subscale, $\mathrm{F}(2,18)=29.06, p<0.001, \eta^{2}=$ 0.605 , and on the anxious subscale, $\mathrm{F}(2,18)=65.83, p<0.001, \eta^{2}=0.776$. The pair-wise comparisons showed significant decreases (all $p<0.01$ ) for both scales between all measurement times.

\section{Discussion}

Depressive disorders are among the most common psychiatric disorders (Essau et al., 1999), but despite the importance of this clinical picture, $70 \%$ to $80 \%$ of depressed adolescents still remain untreated (Cicchetti \& Toth, 1998). The prevalence of anxiety disorders is substantial (Merikangas et al., 2010). In addition, there is a high comorbidity between depression and anxiety symptoms (Hinden et al., 1997; Merikangas et al., 2010). Psychodynamic therapy, which works with insight into conflicts and focuses on problems and stress in close relationships, is particularly suitable for these patients. In fact, psychodynamic therapy in adolescents has historically a long tradition (Freud, 1958) and is offered frequently (Midgley et al., 2017). Looking at research, however, it is surprising that there are only a limited number of studies that assess the efficacy of psychodynamic therapy in this age group.

Two recent reviews on the state of research still indicate a high demand for psychodynamic psychotherapy studies (Midgley \& Kennedy, 2011; Midgley et al., 2017). The recent meta-analysis by Weisz et al. (2017) summarizes 50 years of research in this area and includes 447 studies and 30,031 patients. Across all disorder groups, in $63 \%$ of the cases, the patients in the treatment group improved more than in the untreated control group. This particularly affects patients with anxiety disorders; the effects for patients with depression or comorbid disorders were less good. The multilevel meta-analysis showed an overall effect size of $\eta^{2}=0.45$, which also decreased slightly after the 11-month follow up 
to $\eta^{2}=0.39$. Together the authors concluded a medium effect that was relatively permanent. However, it should be noted that only $30 \%$ of the patients were adolescents, the treatment duration was relatively short (15 hours in 15 weeks) and only a very small group of psychodynamic therapies were included.

This study therefore sets out to evaluate whether psychodynamic treatment is working in adolescents, which effectively reduces psychopathology. We focused on two frequent diagnoses: F32 and F40. The adolescent patients participated in psychodynamic treatment with 1 hour per week over a time of approximately two years (mean duration 86 hours). The treatment focuses on coping with intrapsychic conflicts, the regulation of negative emotions and the perception of self and others in social interactions. Accordingly, the therapy goals are more extensive and the reduction of symptoms is only one of the treatment goals. Nevertheless, the reduction of the symptoms is important, because they cause considerable suffering not only for the affected youth, but also for their families. In the parents' assessment, psychodynamic treatment achieved the goal of symptom reduction. The parents' report of symptom reduction from the beginning to the end of psychodynamic therapy showed moderate to high effects (ranging between $\eta^{2}=0.40$ and 0.78 ), demonstrating the effectiveness of this form of psychotherapy for adolescents. Overall, the effects were higher than in the meta-analysis of Weisz et al. (2017) and consistent over both disorders, F30 and F42. In addition, anxiety symptoms of depressed patients were reduced especially in the later course of therapy, suggesting that a long-term treatment is useful. As a rule, psychodynamic therapy includes several phases, in which the first phase consists primarily of establishing the therapeutic relationships and developing a working alliance, while in the middle phase, work focuses on the underlying problems, and in the last phase, the gains that have been achieved are generalized to diverse situations. Anxiety symptoms are symptoms that develop early in life, and it is possible that basic trust must first be built up in the therapeutic relationship before working on the underlying conflicts and relationship difficulties is possible.

Affective disorders such as depression and anxiety disorders are among the central disorders in adolescence. They pose a significant health risk, not only because of the increased suicide rate. Of note, stabilities of untreated mood disorders are high with symptom persistence of $60 \%$ to $75 \%$ found in anxiety disorders and depression. Although this study was carried out in a naturalistic setting and refrained from a control group, the effects from the perspective of the parents are remarkable. This underlines that adolescents with these disorders benefit greatly from long-term therapies of psychodynamic origin. A follow up is planned in order to establish the stability of the effects.

\section{Limitations and Recommendations for Future Research}

A major limitation of the study is resulting from not having a comparison group.

One could argue that parents simply become more optimistic over time or that 
some unknown confounding variables has led adolescents to improve over time. Also, one could argue that the symptoms grow out over time, since adolescent development may lead to progressive changes and the symptoms thus could disappear. However, it is very unlikely that depression and anxiety symptoms on a clinical level may disappear without treatment. On the contrary, untreated anxiety disorder and depression on a clinical level have a high stability to continue to adulthood (Cicchetti \& Toth, 1998). The choice of the control group is also an ethical problem, because one should not leave such disorders, which cause great suffering, untreated. Some researchers work with a waiting control group, i.e. the control group is treated about 6 months after the treatment group. This possibility was not useful in our study, since the interval of 6 months is not comparable with the effects after 2 years. Although the absence of the control group is a major shortcoming, this does not completely invalidate our results.

A further shortcoming is the reliance on only one respondent, as studies have shown discrepancies in the view of adolescents and their parents (Achenbach, McConaughy, \& Howell, 1987). In the meta-analysis by Weisz et al. (2017), respondent effects were found: parents reported less effects than the patients themselves when it came to depression. This suggests that the effect strengths may be even greater if the youth self-report would be included. It is therefore recommended that in future studies, the adolescent self-report should be assessed, too. In addition, due to the high percentage of depression and anxiety on a subclinical level, future studies should deal with these symptoms, as they can be partly considered as precursors of later clinically relevant depression and anxiety. School based programs for these adolescents can be helpful (Weisz, Krumholz, Santucci, \& Ng, 2015). Due to the gender differences in depression, future studies could also profit from analyzing gender specific effects in psychodynamic treatment over time. Further, we excluded patients showing comorbid diagnosis of F32 and F40. Keeping in mind that these cases are rather rare, future studies should investigate therapeutic efficiency on patients with both disorders in order to draw an image close to reality.

\section{Funding}

The study received no funding.

\section{Ethical Approval}

All procedures performed in studies involving human participants were in accordance with the ethical standards of the institutional and/or national research committee and with the 1964 Helsinki declaration and its later amendments or comparable ethical standards.

\section{Conflicts of Interest}

The author declares that she has no conflict of interest. 


\section{References}

Achenbach, T. M., McConaughy, S. H., \& Howell, C. T. (1987). Child/Adolescent Behavioural and Emotional Problems: Implications for Cross-Informant Correlations for Situational Specifity. Psychological Bulletin, 101, 213-232. https://doi.org/10.1037/0033-2909.101.2.213

Achenbach, T., \& Edelbrock, C. (1983). Behavioral Problems and Competencies Reported by Parents of Normal and Disturbed Children Aged Four Through Sixteen. Monographs of the Society for Research in Child Development, 46, Serial No. 188. https://doi.org/10.2307/1165983

Benjet, C., Georgiades, K., \& Swendsen, J. (2010). Lifetime Prevalence of Mental Disorders in US Adolescents: Results from the National Comorbidity Study Adolescent Supplement (NCS-A). Journal of the American Academy of Child and Adolescent Psychiatry, 49, 980-989. https://doi.org/10.1016/j.jaac.2010.05.017

Chorpita, B. F., Daleiden, E. L., Ebesutani, C., Young, J., Becker, K. D., Nakamura, B. J., Phillips, L., Ward, A., Lynch, R., Trent, L., Smith, R. L., Okamura, K., \& Starace, N. (2011). Evidence-Based Treatments for Children and Adolescents. Clinical Psychology Science and Practice, 18, 154-172. https://doi.org/10.1111/j.1468-2850.2011.01247.x

Cicchetti, D., \& Toth, S. L. (1998). The Development of Depression in Children and Adolescents. American Psychologist, 53, 221-241. https://doi.org/10.1037/0003-066X.53.2.221

Costello, E. J., Foley, D. L., \& Angold, A. (2006). 10-Year Research Update Review: The Epidemiology of Child and Adolescent Psychiatric Disorders: II. Developmental Epidemiology. Journal of the American Academy of Child \& Adolescent Psychiatry, 45, 8-25. https://doi.org/10.1097/01.chi.0000184929.41423.c0

Döpfner, M., Berner, W., \& Lehmkuhl, G. (1995). Reliabilität und faktorielle Validität des Youth Self-Report und der Child Behavior Checklist bei einer klinischen Stichprobe. Diagnostica, 41, 221-244.

Essau, C. A., Conradt, J., \& Petermann, F. (1999). Frequency and Comorbidity of Social Phobia and Social Fears in Adolescents. Behaviour Research and Therapy, 37, 831-843. https://doi.org/10.1016/S0005-7967(98)00179-X

Ezpelata, L., \& Domènech, A. A. (2006). A Comparison of Pure and Comorbid CD/ODD and Depression. Journal of Child Psychology and Psychiatry, 47, 704-712. https://doi.org/10.1111/j.1469-7610.2005.01558.x

Fonagy, P., Roth, A., \& Higgitt, A. (2005). The Outcome of Psychodynamic Psychotherapy for Psychological Disorders. Clinical Neuroscience Research, 4, 367-377. https://doi.org/10.1016/j.cnr.2005.03.005

Freud, A. (1958). Adolescence. Psychoanalytic Study of the Child, 13, 255-278. https://doi.org/10.1080/00797308.1958.11823182

Herbert, J. D., Gaudiano, B. A., Rheingold, A. A., Moitra, E., Myers, V. H., Dalrymple, K. L., \& Brandsma, L. L. (2009). Cognitive Behavior Therapy for Generalized Social Anxiety Disorder in Adolescents: A Randomized Controlled Trial. Journal of Anxiety Disorders, 23, 167-177. https://doi.org/10.1016/j.janxdis.2008.06.004

Hinden, B. R., Compas, B. E., Howell, D. C., \& Achenbach, T. M. (1997). Covariation of the Anxious-Depressed Syndrome during Adolescence: Separating Fact from Artifact. Journal of Consulting and Clinical Psychology, 65, 5-14. https://doi.org/10.1037/0022-006X.65.1.6

Liu, F. F., \& Adrian, M. C. (2019). Is Treatment Working? Detecting Real Change in the Treatment for Child and Adolescent Depression. Journal of the American Academy of 
Child and Adolescents Psychiatry, 58, 1175-1164. https://doi.org/10.1016/j.jaac.2019.02.011

McCarty, C., \& Weisz, J. R. (2007). Effects of Psychotherapy for Depression in Children and Adolescents. Journal of the American Academy of Child and Adolescent Psychiatry, 46, 879-886. https://doi.org/10.1097/chi.0b013e31805467b3

Merikangas, K. R., He, J. P., Burstein, M., Swanson, S. A., Avenevoli, S., Cui, L., Benjet, C., Georgiades, K., \& Swendsen, J. (2010). Lifetime Prevalence of Mental Disorders in US Adolescents: Results from the National Comorbidity Study-Adolescent Supplement (NCS-A). Journal of the American Academy of Child and Adolescent Psychiatry, 49, 980-989. https://doi.org/10.1016/j.jaac.2010.05.017

Midgley, N., \& Kennedy, E. (2011). Psychodynamic Psychotherapy for Children and Adolescents: A Critical Review of the Evidence Base. Journal of Child Psychotherapy, 37, 232-260. https://doi.org/10.1080/0075417X.2011.614738

Midgley, N., O’Keeffe, S., French, L., \& Kennedy, E. (2017). Psychodynamic Psychotherapy for Children and Adolescents: An Updated Narrative Review of the Evidence Base. Journal of Child Psychotherapy, 43, 307-329. https://doi.org/10.1080/0075417X.2017.1323945

Polanczyk, G. V., Salum, G. A., Sugaya, L. S., Caye, A., \& Rohde, L. A. (2015). Annual Research Review: A Meta-Analysis of the Worldwide Prevalence of Mental Disorders in Children and Adolescents. Journal of Child Psychology and Psychiatry, 56, 345-365. https://doi.org/10.1111/jcpp.12381

Shirk, S. R., Kaplinski, H., \& Gudmundsen, G. (2009). School-Based Cognitive-Behavioral Treatment for Adolescent Depression. Journal of Emotional and Behavioral Disorders, 17, 106-117. https://doi.org/10.1177/1063426608326202

Szigethy, E., Kenney, E., Carpenter, J., Hardy, D. M., Fairclough, D., Bousvarus, A., Keljo, D., Weisz, J., Beardslee, W. R., Noll, R., \& DeManso, D. R. (2007). Cognitive-Behavioral Therapy for Adolescents with Inflammatory Bowel Disease and Subsyndromal Depression. Journal of the American Academy of Child \& Adolescent Psychiatry, 46, 1290-1298. https://doi.org/10.1097/chi.0b013e3180f6341f

Weisz, J. R., \& Jensen, A. L. (2001). Child and Adolescent Psychotherapy in Research and Practice Contexts: Review of the Evidence and Suggestions for Improving the Field. European Child and Adolescent Psychiatry, 10, 12-18. https://doi.org/10.1007/s007870170003

Weisz, J. R., Jensen-Doss, A., \& Hawley, K. M. (2005). Youth Psychotherapy Outcome Research: A Review and Critique of the Evidence Base. Annual Review of Psychology, 56, 337-363. https://doi.org/10.1146/annurev.psych.55.090902.141449

Weisz, J. R., Krumholz, L. S., Santucci, K. T., \& Ng, M. Y. (2015). Shrinking the Gap between Research and Practice: Tailoring and Testing Youth Psychotherapies in Clinical Care Contexts. Annual Review of Clinical Psychology, 11, 139-163. https://doi.org/10.1146/annurev-clinpsy-032814-112820

Weisz, J. R., Kuppens, S., Eckshtain, D., Ugueto, A. M., Hawley, K. M., \& Jensen-Doss, A. (2017). Do Evidence-Based Youth Psychotherapies Outperform Usual Clinical Care? A Multilevel Meta-Analysis. JAMA Psychiatry, 70, 750-761.

https://doi.org/10.1001/jamapsychiatry.2013.1176 